\title{
PRESHEATH PROFILES IN SIMULATED TOKAMAK EDGE PLASMAS
}

\author{
B. LaBombard, R.W. Conn, Y. Hirooka, R. Lehmer, \\ W.K. Leung, R.E. Nygren, Y.Ra, G. Tynan
}

UCLA $\mid \mathrm{PPG}-1147$

April, 1988

\section{DISCLAIMER}

\begin{abstract}
This report was prepared as an account of work sponsored by an agency of the United States Government. Neither the United States Government noz any agency thereof, nor any of their employees, makes any warranty, express or implied, of assumes eny legal liability of responsibility for the accuracy, completeness, or usefulness of ary information, apparatus, product, or process disclosed, or represents that its use would nat infringe privalely owned rights. Reference herein to any specific commercial product, process, or service by trade name, trademark, manufacturer, or otherwise does not necessarily constitute or imply its endorsement, recommendation, or fayoring by the United States Government or any agency thereof. The views and opinions of authors expressed herein do not necessarily state or reflect those of the United States Government or any agency thereot.
\end{abstract}

Department of Mechanical, Aerospace and Nuclear Engineering Institute of Plasma and Fusion Research University of California, Los Angeles, CA, 90024, U.S.A. 


\title{
PRESHEATH PROFILES IN SIMULATED TOKAMAK EDGE PLASMAS
}

\author{
B. LABOMBARD, R.W, CONN, Y. HIROOKA, R. LEHMER, \\ W.K. LEUNG, R.E. NYGREN, Y. RA, G. TYNAN \\ Institute of Plasma and Fusion Research \\ Department of Mechanical, Aerospace and Nuclear Engineering \\ University of Califomia, Los Angeles \\ Los Angeles, CA 90024, USA
}

The PISCES plasma surface interaction facility at UCLA generates plasmas with characteristics similar to those found in the edge plasmas of tokamaks ( $\mathrm{n}=10^{11}-10^{13} \mathrm{~cm}^{-3}, \mathrm{Te}_{\mathrm{e}}=3$ - $30 \mathrm{eV}$ ). Steady state magnetized plasmas produced by this device are used to study plasma-wall interaction phenomena which are relevant to tokamak devices. We report here progress on some detailed investigations of the presheath region that extends from a wall surface into these "simulated tokamak" edge plasma discharges along magnetic field lines.

A number of edge plasma diagnostics have been specially developed for this work and are now becoming fully operational including a fast-scanning multiple Langmuir/Emissive/Mach probe system and a CID camera imaging system. Both systems allow detailed diagnoses of a $10 \times 10 \times 10$ $\mathrm{cm}^{3}$ region of magnetized plasma in contact with a wall surface. Measurements of plasma density, electron temperature, floating potential, space potential, and bulk plasma flow velocities have been obtained in hydrogen, helium, and argon plasmas with densities ranging from $10^{12} 1010^{13} \mathrm{~cm}^{-3}$, electron temperatures from 5 to $15 \mathrm{eV}$, and axial magnetic fields of 0.2 to $1.4 \mathrm{kG}$. Plasma density profiles along the magnetic field typically show a characteristic factor of 2 decrease towards the wall surface. A plasma potentiai variation in the near presheath zone of order $0.5 \times \mathrm{T}_{\mathrm{e}}$ is measured, consistent the bulk plasma flow approaching the ion sound speed near the wall surface as inferred from a simple "free fall" model. A Boltzmann model for the presheath density profile accurately tracks the density profile measured both by the Mach probe and by spectroscopic means. Flow profiles are used as a consistency check on various magnetized Mach probe theories. Results suggest that cross-field transport of parallel momentum through ion viscosity is relatively unimportant in PISCES discharges. In discharges that have a non-thermal "hot" electron component with a number density of a few percent or more of the "colder" bulk electrons, axial profiles of space potential and floating potential indicate that the overall electron distribution function at the wall surface is "hotter". This is found to be consistent with an "energy filtering" model in which a fraction of the colder, bulk electrons is reflected by the presheath potential drop while the "hotter" electrons are able to reach the sheath edge near the wall surface. 


\section{Introduction}

The presheath zone is loosely defined as the quasineutral plasma region extending from the sheath near a wall surface into the bulk plasma. In a magnetic field, the presheath zone extends large distances along magnetic field lines and is populated by plasma through cross-field transport and/or ionization processes. Thus, an entire tokamak scrape-off layer can be considered as a large magnetized presheath plasma zone. Electric fields within the presheath are responsible for accelerating the ion species from rest within the bulk plasma to sound speed velocities at the sheath edge thereby satisfying the Bohm criterion.

A detailed understanding of presheath physics is important not only for assessing the overall impact of plasma/wall interactions on a tokamak discharge but also in diagnosing the tokamak edge plasma itself. Local wall erosion/redeposition rates depend critically on the presheath electric fields and plasma flows which can sweep ionized impurities back onto wall surfaces. The interpretation of data collected by an electric probe in such a sowing plasma relies on an accurate model of not only the plasma presheath zone in which it is immersed but also the small presheath zone that is induced by the probe surface itself.

The PISCES plasma surface interaction facility at UCLA [1,2] provides a means for studying tokamak-like edge plasma-wall interaction phenomena from both the materials and plasma physics points. We report in this paper on the progress of some basic plasma physics experiments in PISCES aimed at diagnosing in detail a magnetized presheath region of a "simulated tokamak" plasma-wall interaction zone. The goals of these experiments are threefold: 1) to characterize the presheath zone, providing a more accurate interpretation of plasma-wall interaction experiments, 2) to assemble an experimental data base to enable a test of various theoretical plasma physics models of the presheath zone in "tokamak-like" plasmas, and 3) to test the interpretation of electric probe diagnostics in a flowing plasma. This paper focuses primarily on progress made in achieving the first goal. Work towards the latter two goals is ongoing and will be reported in future publications [3],

\section{Experimental Arrangement}

The PISCES facility produces plasma by a PIG discharge using a hot $\mathrm{LaB}_{6}$ cathode disc. Details of the plasma source operation and construztion can be found in the references $[1,2,4]$. The disc cathode and cylindrical anode are placed at one end of a vacuum chamber which is coaxial with a series of magnetic field coils at Helmholzz spacing. The axial magnetic field provides radial 
plasma confinement and maps from a hot cathode disc onto an electrically floating end plate (fig.1). Electrons emined from the hot $\mathrm{L}_{\mathrm{a}} \mathrm{B}_{6}$ disk are accelerated by the cathode-plasma sheath drop and injected along magnetic field lines with energies in the range of 60 to $150 \mathrm{eV}$. The sheath at the electrically floating end plate reflects most of the electrons back to the cathode and allows these particles to efficiently ionize the background fill gas $\left(5 \times 10^{-5}\right.$ to $5 \times 10^{-3}$ torr) even though their mean free path can be much longer than the column length. Steady-state plasmas with densities in the range of $10^{11}$ to $10^{13} \mathrm{~cm}^{-3}$ and electron temperatures of 3 to $30 \mathrm{eV}$ are readily achieved in a 6 to 10 $\mathrm{cm}$ diameter cylindrical column of approximately $100 \mathrm{~cm}$ in length. Similar to a tokamak edge plasma, these plasmas exhibit fully "turbulent" dynamics, namely, broadband fluctuation spectra and cross-field particle diffusion coefficients that are characteristic of the Bohm level. In the standard operation, material samples are immersed in the plasma column for testing through a modified end plate, as shown in fig. 1.

The arangement for the presheath plasma profile measurements reported here is also shown in fig. 1. A test sample is inserted into the plasma and allowed to electrically "float", drawing no net current from the plasma. A zone of plasma of approximately $10 \times 10 \times 10 \mathrm{~cm}^{3}$ in front of the material test sample is investigaeed by three principal diagnostics: a fast-scanning pneumatically driven probe; a stationary water-cooled Langmuir probe; and a solid state CID (charge injected device) camera. Since the Debye length is typically much less than $100 \mu \mathrm{m}$, these three diagnostics record conditions in the quasineutral presheath plasma zone in front of the material sample.

A fast-scanning probe system capable of employing a wide variety of electrical probe techniques and geometries has been specially built to diagnose PISCES plasmas [5]. A pneumatic cylinder is used to drive a single or multiple probe tip across the plasma column and back ( $15 \mathrm{~cm}$ stroke) in less than $\mathbf{4 0 0} \mathrm{msec}$. This enables a vertical profile to be taken in one "shot" and at the same time limits the total energy deposited on the probe to safe levels during the high density plasmas that can be achieved in PISCES (power fluxes $>400 \mathrm{~W} / \mathrm{cm}^{2}$ ). A differentially-pumped sliding seal allows the probe to be positioned for a fast vertical scan at various axial distances from the sample surface. By vertically scanning the plasma column through its centerline at uniformly spaced axial locations, a complete map of plasma parameters in the near presheath of the material sample can be assembled.

A unique probe tip that functions simultaneousiy as a two-sided Langmuir probe (or "Mach probe") and as an emissive probe was constructed for these and other experiments in PISCES. The probe tip simply consists of a $4.8 \mathrm{~mm}$ diameter 4-hole extruded alumina rod with a specially sculptured end (fig. 2). Two $1 \mathrm{~mm}$ diameter molybdenum wires of approximately $2.5 \mathrm{~mm}$ of exposed length collect particles on opposing sides of an alumina separator. The $45^{\circ}$ angle on the 
alumina allows the probe to be operated as a unidirectional probe or as a bidirectional probe, depending on its orientation with respect to the magnetic field. For the presheath measurements repored here, the probe was operated as a bidirectional or "Mach probe" with the "up-stream" probe facing towards the $\mathrm{LaB}_{6}$ cathode along magnetic field lines and the "down-stream" probe facing the material test sample. Both probes were continuously biased in ion saturation at -140 volts. Ion saturation current data were numerically smoothed over a $10 \mathrm{msec}$ time window to reduce "noise" due to density fluctuations, (typically $\delta n / n \approx 0.3$ to 0.5 ).

A $0.25 \mathrm{~mm}$ diameter $1 \%$ thoriated tungsten wire loop was inserted into the remaining two holes of the alumina and employed as a thermionic electron emitter. Typically, the tungsten filament was preheated by an AC current for approximately $0.5 \mathrm{sec}$ before the probe initiated its fast vertical scan. The filament was maintained in a mode of strong electron emission ( $T>2000$ ${ }^{\circ} \mathrm{C}$ ) throughout the inward travel of the probe. Power was turned off to the filament on the retum stroke and the entire probe assembly was allowed to cool between scans. The "floating" voltage of the filament was recorded and interpreted as the local plasma potential in the case when the filament strongly emitted electrons and as the usual plasma floating potential in the case when the filament was turned off during a scan. A stationary, water-cooled Langmuir probe could also be inserted into the plasma stream and was typically used to cross-check data from the fast-scanning probe or to record a complete Langmuir characteristic at a fixed spatial position.

An image intensified CID camera was used during these experiments to record lineintegrated brightness profiles of $\mathrm{H}_{\alpha}(6563 \AA)$ emission as well as to record a picture of the experimental arrangement on video tape for later reference (fig.1). Since the mean free paths of both atomic and molecular hydrogen in PISCES plasmas are typically much larger than the plasma radius, a relatively uniform hydrogen neutral density can be maintained within the diagnosed presheath region. In non-hydrogen discharges this was accomplished by "bleeding in" trace amounts of hydrogen into the feed gas. Under these conditions, the variation in $\mathrm{H}_{\mathbf{\alpha}}$ emissivity along a given magnetic field line was expected to track the local electron density variation in the presheath as long as the electron temperature was constant.

In order to obtain the local $\mathrm{H}_{\boldsymbol{\alpha}}$ emissivity as a function of both plasma radius and distance along magnetic field lines, video images were digitized and an Abel inversion was performed ón the cylindrically symmetric brightness data. Profiles of $\mathrm{H}_{\alpha}$ emissivity were then compared to density profiles measured independently by the fast-scanning probe. This was used as a crosscheck on the probe data to assure that the insertion of the probe did not significantly perturb the presheath plasma profiles. 


\section{Results}

\subsection{Presheath Density and Potencial Profiles}

Fig. 3 shows a typical mapping of plasma density and potential in the presheath plasma zone from a series of fast probe scans. These data were obtained in a helium discharge with the following parameters: helium fill pressure (measured near the material sample) $\mathrm{P}_{\mathrm{He}}=4 \times 10^{-3}$ torr, electron temperature on axis $T_{e}=12 \mathrm{eV}$, and axial magnetic field $B=1.4 \mathrm{kG}$. The mapping was obtained by scanning the fast probe twice in the vertical $(x)$ direction at each of 22 regularly spaced positions in the horizontal $(z)$ direction. The two vertical scans at each horizontal position allowed the emissive probe to altemately record floating potential profiles as a cold filament and plasma potential profiles as a hot, strongly emitting filament. The Mach probe was operated in ion saturation mode for all scans.

The density reported in fig. 3(a) was estimated by simply averaging the ion saturation. currents collected on the up-stream and down-stream facing probes and assuming that the electron temperature had a spatially uniform value of $12 \mathrm{eV}$. This technique effectively treats the two probes as one larger probe. The electron temperature was measured by momentarily inserting a watercooled Langmuir probe at an axial position of $z=4 \mathrm{~cm}$. Since the vertical electron temperature profile is not unfolded from the data in fig. 3 , the density variation in the vertical direction should not be taken as completely accurate. However, in this discharge the electron temperature is found to be very nearly constant along the magnetic field. Consequently, the relative variation in density along the $z$ axis in fig. 3(a) is reliable. Note that there is nearly a factor of two drop in the density over an $8 \mathrm{~cm}$ distance along the magnetic field lines that intercept the material sample.

Plasma potential contours shown in fig. 3(b) were deduced from the potential acquired by the hot tungsten filament. At filament temperanures near $2000^{\circ} \mathrm{C}$, the thermionic electron flux can approach and exceed the random electron flux in the plasma. Under these conditions, the potential of the filamen+ tends to "float" to a value that is close to the local plasma potential. Power to the filament was adjusted so as to achieve this condition of strong electron emission by incrementally increasing the $\mathrm{AC}$ voltage on the filament until the potential profile recorded by the probe no longer changed.

Fig. 3(b) shows that vertical plasma potential profiles exhibit a minimum on the central axis of the plasma column $(x=0)$. The steepest gradients in potential occur in the vertical direction. Radial electric fields on the order of 12 volts/ $\mathrm{cm}$ are estimated from this potential variation. In the axial direction, there is evidence of a weaker potential variation along field lines intercepted by the material sample. This is the presheath electric field induced by the presence of the material sample. 
A potential variation on the axis of approximately 6 yolts occurs over a distance of $8 \mathrm{~cm}$, consistent with the expected order of magnitude for the presheath potential variation of $0.5 \times T_{e}$.

Due to the anode/cathode geometry which is used to form the PISCES plasma (see fig. 1), a strong radial electric field is always present. The radial electric field in PISCES plasmas appears to adjust itself for a given axial magnetic field so as to maintain azimuthal ExB rotation speeds that are from 0.1 to 1.0 times the ion sound speed. Such strong azimuthal rotation velocities are believed to contribute to the plasma turbulence and Bohm-like cross-field transport fluxes observed in PISCES. However, this azimuthal velocity profile also complicates the interpretation of any Langmuir probe measurements which are not made on the central axis of rotation. Indeed, the vertical density asymmetry shown in fig. 3(a) can be simply attributed to a data interoretation problem stemming from the effect of the azimuthai ExB rotation. In these plasmas the ExB azimuthal flow was directed into the top half of fig. 3(a) and out the bottom half. Referring to fig. 2 , one can see that the "sculptured" probe tip was orientated in such a way as to face into this flow during the upper half of its vertical scan and away from the flow during the bottom half of its scan. As a result, a relatively higher ion saturation flux could be collected while the probe was in the upper half of the scan. The true vertical density profile is therefore better represented by an average of the upper and lower half values of fig. 3(a). In any event, the remainder of this paper focuses on axial profiles along $x=0$ where the effect of this ExB rotation on data interpretation is expected to be minimal.

Ion saturation currents recorded on the up-stream facing probe were found to typically exceed those recorded on the down-stream facing probe by a factor of two or more, suggesting the presence of a strong parallel plasma flow directed towards the material sample. Even with the Mach probe rotated $180^{\circ}$ with respect to the magnetic field, the same ratio of up-stream facing to downstream facing flux was obtained indicating that this ratio was not simply due to a difference in probe collection areas. Nevertheless, rather than attempt to account for a parallel plasma flow in computing the local density, an equivalent "no flow" plasma density is computed from standard planar probe theory. In this way, the data could be displayed independent of the errors that might be introduced by trying to model the effect of the flow.

"Densities" deduced from the flux on the up-stream facing probe, the flux on the downstream facing probe, and an average of these two fluxes, assuming no plasma flow, are plotted versus axial position in fig. 4. Also plotted in fig. 4 are an $\mathrm{H}_{\alpha}$ emissivity profile inferred from the CID camera, and a plasma density profile inferred from a Boltzmann law, $n(x)=n_{0} \exp \{(\Phi(x)$ $\left.\Phi_{0}\right) / T_{e}$ \}, using the measured values of the plasma potential and electron temperanure. Since the latter two profiles do not yield an absolute value for the plasma density, they are both normalized to the "average" density profile obtained from the Mach probe at an axial position of $8 \mathrm{~cm}$. Helium 
plasma conditions for this data set were: $P_{H e}=2 \times 10^{-3}$ torr, $T_{e}=8 \mathrm{eV}$, and $B=0.8 \mathrm{kG}$. A hydrogen partial pressure of $\mathrm{P}_{\mathrm{H}_{2}}=4 \times 10^{-5}$ torr was added in order to allow $\mathrm{H}_{\alpha}$ profiles to be recorded in this plasma

A number of important points are illustrated in fig. 4. Since the emissive probe measures the plasma potential by effectively cancelling the net random electron flux to the probe, bulk plasma flow velocities on the order of the ion sound speed should not influence this measurement. Consequently, the density profile inferred from the Boltzmann law can be taken as the true presheath plasma density variation. The "average" density from the Mach probe fits the Boltzmann density profile very well, somewhat justifying the ad hoc technique of averaging the up-stream and down-stream ion saturation currents. Normalization of the Boltzmann density profile to the upstream "density" or down-stream "density" profiles do not yield such a good fit.

The $\mathrm{H}_{\alpha}$ emissivity profile also tracks the "average" density from the Mach probe. Again, normalization of the $\mathrm{H}_{\alpha}$ emissivity profile to the up-stream "density" or down-stream "density" profiles only yields a poorer fit. The $H_{\alpha}$ emissivity profile was recorded without any probes inserted into the plasma. Thus, it indicates that the presheath plasma density profiles are not significantly perturbed by the insertion of the fast probe.

Finally, these experiments point out that caution should be exercised in deducing the local plasma density from a directional Langmuir probe in the presence of a plasma flow. Without information from the down-stream facing probe, one could easily overestimate plasma densities by $50 \%$ or more, by incorrectly assuming that the plasma flow speed is zero.

\subsection{Presheath PIasma Flows}

A number of models have been considered in an attempt to predict plasma flow velocities from magnetized Mach probe measurements [6-9]. In these models, the plasma flow velocity along magnetic field lines, expressed in Mach number (M), is related to the ratio (R) of plasma flux collected on the up-stream facing probe over the flux collected on the down-stream facing probe. This functional reiationship, $M=f(R)$, varies significantly from model to model and depends on the physics that is emphasized in each.

Fig. 5 shows Mach number profiles computed from three different models considered by Stangeby [7], Harbour and Proudfoot [6], and Hutchinson [8], using the probe data that was shown in fig. 4. Positive Mach numbers refer to parallel plasma flow velocities directed towards the material sample. Also shown in fig.5 are Mach numbers based on a "free fall" velocity of ions 
accelerated by the presheath potential drop. These "free fall" profiles were derived from the simple conservation of energy formula,

$$
M(x)=\sqrt{M_{0}^{2}+\frac{2\left(\Phi_{0}-\Phi(x)\right)}{T_{\theta}}}
$$

using the measured values for the presheatri potential profile. The initial velocities for each "free fall", M0, were chosen to coincide with the velocities predicted from each of the three models at $z=$ $8 \mathrm{~cm}$.

Although eq. (1) is not rigorously correct, it predicts the maximum change in plasma flow velocity for a given potential drop within the presheach region (assuming ion pressure effects are negligible). Effects such as ionization, charge exchange, cross-field particle transport, and viscosity only serve to reduce this flow speed by effectively adding drag. Fig. 5 shows that, independent of the initial velocity of the flow at $\mathrm{z}=8 \mathrm{~cm}$, the "free fall" Mach number approaches 1 near the material sample surface. This is consistent with the Bohm criterion which requires that the flow be greater than or equal to Mach 1 at the wall surface. It also justifies the use of a "free fall" model to approximate the plasma flow profile since the introduction of any appreciable drag effects would not allow the flow to achieve Mach 1 at the wall.

A Mach number profile deduced from the measured flux ratio, R, and the Stangeby model [7] compares most favorably with the "free fall" velocity profile. The Harbour and Proudfoot [6] and Hutchinson [8] models predict much lower flow speeds everywhere and seem to sufier from the rather severe problem of not achieving a Mach 1 flow near the material sample surface. This is particularly true of the Hutchinson model and may indicate that cross-field parallel momentum transfer due to ion viscosity is relatively unimportant in these discharges [9]. In fact, one expects that the "correct" model should predict Mach numbers greater than 1 at the wall surface using the measured flux data. As the Mach probe gets very close to the wall, the flux tube from which the down-stream facing probe collects particles becomes cut-off by the wall. As a result, less flux should be collected by this probe near the wall surface and hence erroneously large flux ratios recorded there. Mach numbers deduced from these overestimated flux ratios near the wall sufface should therefore be higher than actual Mach numbers. Nevertheless, only the Stangeby model is found to exhibit this trend, estimating Mach numbers greater than 1 at the wall,

To be consistent in the analysis of Mach probe data, one needs to account for the local flow velocity in determining the local plasma density. Fig. 6 plots the density profile data displayed in Iig. 4 , corrected for the plasma flows inferred from each of the three models. All three models 
predict lower plasma densities near the material sample than is inferred from the Boltzmann law. However, this may simply be a consequence of the down-stream facing probe collecting less particles as its flux tube becomes interrupted by the sample surface. Since the density profile is found to he predicted equally well by all three models, no further information about the applicability of these models for the analysis of PISCES plasmas is obtained.

\subsection{Non-Maxwellian Electron Effects}

The power input to PISCES discharges originates from "primary electrons" injected from the hot $\mathrm{LaB}_{6}$ cathode. Under the some conditions, the plasma-cathode sheath potential drop can be as high as $\mathbf{1 5 0}$ volts. If at the same time the plasma density is low, thermalization of these high energy electrons becomes poor and the electron distribution function displays a non-thermal high energy component. From analysis of Langmuir probe characteristics, it is found that these nonthermal electron distribution functions can be accurately described by a two temperature distribution model. The "cold" or bulk component of the electrons typically makes up $90 \%$ or more of the electron population while the "hot" component, with an equivalent tenperature in the range of 30 to $60 \mathrm{eV}$, makes up the balance of the electron population.

One consequence of a two temiparature electron distribution function in PISCES is that the presheath density profile is coupled to the potential profile through a modified Boltzmann law,

$$
n(x)=n_{0}^{\text {ool }} \exp \left\{\left(\Phi(x)-\Phi_{0}\right) / T_{0}^{\text {cold }}\right\}+n_{0}^{\text {hot }} \exp \left\{\left(\Phi(x)-\Phi_{0}\right) / T_{0}^{\text {hor }}\right\}
$$

The effect of a presheath potential drop of 0.5 to 0.7 times the bulk or "cold" electron temperature is to reduce the density of the cold electron component near the wall to approximately half its value far from the wall surface. On the other hand, the hot component is relatively unaffected by the potential change and maintains a nearly constant density up to the sineath edge at the wall surface. Thus, even in these non-Maxwellian discharges, analysis of density profiles using an isothermal Boltzmaru law (section 3.1) is still approximately valid. However, the effective temperature of the electrons near the wall surface can be "hotter" than the electrons farther upstrean.

Data plotted in fig. 7 shows evidence of a "hotter" electron population near the material sample surface. These data were obtained from the same discharge that was displayed in fig. 3. Langmuir probe characteristics taken at $z=4 \mathrm{~cm}$ indicated a "hot" electron density fraction of approximately $10 \%$ of the bulk with an effective temperature of $30 \mathrm{eV}$ ("cold" component 
temperature was $12 \mathrm{eV}$ ). Two potential profiles from the emissive probe are plotted in fig. 7 one when the filament was cold and one when the filament was hot and strongly emitting electrons. The potential of the cold filament can be simply interpreted as the plasma "floating potenial" while the potential of the hot filament is expected to approximate the local plasma space rotential. In a plasma with Maxwellian electrons at temperature $T_{e}$ and cold ions ( $i_{i} \ll T_{e}$ ), the space potential $(\Phi)$ and floating potential $\left(V_{f}\right)$ are simply relate 1 by

$$
T_{0}=\alpha\left(\Phi-V_{f}\right)
$$

where $\alpha$ takes into account the ratio of ion sound speed to electron themal speed as well as secondary electron emission effects (also geometry effects when the ion Larmor radius is larger than the probe wire diameter as in the case of the emissive probe). For illustration, an electron temperature profile dediced frox eq. (3) is also plotted in fig.7. In order to avoid choosing a value for $\alpha$, the profile is normalized to the electron temperature ("cold" component) inferred 'y the Langmuir probe at $\mathrm{z}=4 \mathrm{~cm}$. This figure clearly shows that the elersion posulation near the material surface is more energetic, causing a depression in the local floating potential relative to the space potential. Of course under these circumstances, $T_{e}$ is not properly dediced from eq. (3) and one must work with the suari iocal distribution function to determine its energy content.

\section{Discussion}

Throughout the aralysis in this paper two critical assumptions have been made about the operation of the inst scanning Mach pmbt, namely, 1) that the probe was large compared to the ion Larmo' radius so that a planar probe theury could be applied and 2) that the perturbotion length of the probe was short relative to the presheati: stali: length in the experiment.

Since the magnetic field strength is relatively low in PISCES, it is recognized that the validity of the first assumpion can beconie marginal even at low ion temperatures. As a result, helium discharges were chosen over hydrogen discharges so as to avoid molecular dissociation processes which can input energy to the ions. From doppler broadening measurements of $\mathrm{He}^{\text {II }}$ emission, $\mathrm{He}^{+}$ions are estimated to have temperatures between 0.5 and $1.2 \mathrm{eV}$ over a wide variety of discharge parameters [10] (unrorrected for any additional broadening effects due to viewing a rotating plasma). $\mathrm{He}^{+}$is expected to be the dominant ion species since the rate of $\mathrm{He}^{++}$formation from $\mathrm{He}^{+}$is almost two orders of magnitude lower than the rate of $\mathrm{He}^{+}$formation from $\mathrm{He}^{0}$ for 
the plasma and neutral conditions in PISCES. At a magnetic field of $0.5 \mathrm{kG}, 0.5 \mathrm{eV} \mathrm{He}^{+}$ions have Larmor radii that are comparable to the Mach probe radius. For this reason, data shown in figs. 3 and 7 were taken in $1.4 \mathrm{kG}$ discharges while the remaining figures show data taken in $0.8 \mathrm{kG}$ discharges. The presheath profiles recorded by the Mach probe were found to be nearly identical for both magnetic field strengths. The only notable difference between runs in $0.8 \mathrm{kG}$ and $1.4 \mathrm{kG}$ was that the current ratio $(R)$ recorded nearest to the material sample was higher for the latter case. This suggests that the perturbation length of the Mach probe can become long enough to interfere with the material sample.

Assuming a Bohm cross-field diffusion rate, the characteristic perturbation length of the Mach probe along the magnetic field $\left(\mathrm{L} \sim \mathrm{a}^{2} \mathrm{Cs} / \mathrm{D}\right)$ is estimated to be approximately $1 \mathrm{~cm}$ for 0.8 $\mathrm{kG}$ and $2 \mathrm{~cm}$ for $1.4 \mathrm{kG}$ B-fields. In this respect, the data taken at $0.8 \mathrm{kG}$ is more reliable. A $1 \mathrm{~cm}$ perturbation length implies that the data shown in figs. 4-6 are the result of "averaging" plasma parameters vver a 1 to $2 \mathrm{~cm}$ axial extent. Since the presheath extends well beyond $8 \mathrm{~cm}$ and has a fairly linear profile, the effect of this spatial "averaging" should not impact the overall results obtained by the Mach probe. However, at distances of less than $2 \mathrm{~cm}$ away from the material sample surface, one expects that Mach probe data should record a profile that depars from the expected trend. Such a departure near the surface is siggested by the data in fig. 5 (Stangeby modei predicting Mach $\$>1$ ) and fig. 6.

\section{Summary}

A number of edge plasma diagnostics have been developed to perform some basic plasma physics experiments in the PISCES facility associated with the formation of a magnetized presheath region near a wall surface. Presheath density, potential, and flow profiles have been deduced under a variety of plasma conditions. Some key observations that have been made from these experiments thus far include:

(1) Density profiles are found 20 exhibit a characteristic factor of 2 decrease along magnetic field lines that intercept a wall surface, consistent with the generally accepted model for the presheath zone.

(2) Plasma potential variations of order $0.5 \times \mathrm{Te}$ are detected in the near presheath region, consistent with density profiles inferred from a Boltzmann law and cross-checked with both "Mach probe" measurements and $\mathrm{H}_{\alpha}$ emissivity profiles.

(3) Plasma flow velocities inferred from "free fall" acceleration of ions using the measured potential profiles are checked against models which predict flow velocities from Mach probe data. 
In all cases the "free fall" model yields flow velocities consistent with a Mach 1 boundary condition at the wall surface. However, Mach probe modeis differ in their predictions of the flow speed. The Stangeby model [7] is found to best fit presheath flow profiles in PISCES plasmas, suggesting that viscosity effects do not play a dominant role [9].

(4) Presheath profiles with a non-Maxwellian electron population are investigated. Low energy electrons are reflected by the presheath potential drop leading to the detection of a "hotter" electron population at the sheath edge near the wall surface.

\section{Acknowledgements}

The authors gratefully acknowledge the invaluable contributions from the PISCES technical staff, especially T. Sketchley, G. Gunner, and J. Elverum for sesistance in hardware and electronics, and $\mathbf{K}$. Andrews and $\mathrm{K}$. Sheedy for computer and data acquisition systems support. Special thanks are extended to $\mathrm{K}$. Chung for both his stimulating discelssions and his assistance in evaluating Mach numbers and densities from the various Mach probe models. This work is supported by the U.S. Department of Energy Contract No. DE-FG03-86ER52134.

\section{References}

[1] D.M. Goebel, D.M., G.A. Campbell, G., R.W. Conn, J. Nucl. Mater. 121 (1984) 277.

[2] D.M. Goebel, and R.W. Conn, J. Nucl. Mater. 128 \& 129 (1984) 249.

[3] Partly in collaboration with L. Hutchinson, B. Lipschultz, and K. Chung at the Massachuserts Institute of Technology, Cambridge Massachusetts, USA.

[4] D. M. Goebel, Y. Hirooka, and T.A. Sketchley, Rev. Sci. Instr. 56 (1985) 1717.

[5] B. LaBombard, R.W. Conn, R. Lehmer, W.K. Leung, Bull. Amer. Phys. Soc. 32 (1987) 1941.

[6] Average of the particle and fluid models considered by P.J. Harbour and G. Proudfoot in analyzing Mach probe data from DITE. See G. Proudfoot, P.J. Harbour, J. Allen, A. 
Lewis, J. Nucl. Mater. $128 \& 129$ (1984) 180 and P.J. Harbour, G. Proudfoot, J. Nucl. - Mater. 121 (1984) 222.

[7] P.C. Stangeby, Phys. Fluids 27 (1984) 2699.

[8] I.H. Hutchinson, Phys. Fluids 30 (1987) 3777.

[9] L.H. Hutchinson, preprint; to be published in Phys. Rev. A.

[10] A. Pospieszczyk, private communication. 


\section{Figure Captions}

Fig. 1 - Experimental arrangement for presheath experiments in the PISCES Plasma Surface Interaction Facility. A PIG discharge is maintained between a hot $\mathrm{LaB}_{6}$ cathode and a cylindrical anode. $10 \mathrm{~cm}$ diameter cylindrical plasmas of $100 \mathrm{~cm}$ length are confined radially by a $0.2-1.4 \mathrm{kG}$ axial magnetic field and guided onto a material test sample. A $10 \times 10 \times 10 \mathrm{~cm}^{3}$ zone of plasma in contact with the marerial sample is investigated in detail by three diagnostics: a fast-scanning probe, a fixed Langmuir probe, and a CD camera.

Fig. 2 - Detail view of fast-scanning probe tip. The probe functions as a two-sided or Mach probe as well as an emissive probe. $1 \mathrm{~mm}$ diameter molybdenum wires collect particles on opposing sides of 4-hole alumina insulator. A loop of thoriated tungsten projects beyond the molybdenum wires, collecting plasma from both directions along the B-field. The loop is resistively heated into strong electron emission over a 1 to $2 \mathrm{sec}$ pulse. The probe tip is pneumatically driven across the plasma in 400 msec.

Fig. 3 - Contour plots of density and potential from the fast-scanning probe. Density profiles exhibit almost a factor of 2 decrease upon approaching the material sample surface along magnetic field lines. The vertical $(x)$ density asymmetry is interpreted as an "artifact" due to a strong aximuthal ExB rotation. The potential profile exhibits large radial E-fields and records a near presheath potential variation of approximately $0.5 \times \mathrm{T}_{e}$ along the central axis.

Fig. 4 - A comparison of density profiles along the central axis from three diagnostics. Three density profiles are inferred from the Mach probe assuming no plasma flow: density from the up-stream facing probe, density from the down-stream facing probe, and an average of these. For comparison, a density profile was computed from the Boltzmann law using the measured potential profile and electron temperature. This profile is shown, normalized to the average density from the Mach probe at $z=8 \mathrm{~cm}$. A density profile inferred from the local $\mathrm{H}_{\alpha}$ emissivity is also plotted with a similar normalization. The consistency in these profiles lends confidence to the experiment and shows the the probe did not perturb the plasma profiles in which it measured. The magnitude of density error bars are suggested by the scarter in these data 
Fig. 5 - Comparison of Mach number profiles deduced from three models. The ratio of up-stream facing flux to down-stream facing flux was used to compute parallel flow speeds from three Mach probe models. For comparison, the "free fall" velocity of ions accelerated by the presheath potential variation is also plotted. The initial velocity of this "free fall" is chosen to coincide with each of the three models at $z=8 \mathrm{~cm}$. The Stangeby model [7] is found to fit the velocity profile daca best and suggests that viscosity does not play a significant role in these discharges [9].

Fig. 6 - Density profiles computed from three Mach probe models, including a correction for plasma flow. For comparison, density profiles computed from a Boltzmann law are overlaid. These profiles are normalized at $z=8 \mathrm{~cm}$. All models predict a lower density at the material sample surface than predicted by the Boltzmann law. This may be a consequence of the Mach probe's flux nube interfering with the material surface.

Fig. 7 - Plasma potential and floating potential profiles showing non-Maxwellian electron effects. Local elecison temperatures computed from the difference in space and floating potentials indicates a "hotter" electron population near the material sample surface in some discharges. The "cold" electron population is reflected by the presheath potential drop while the "hot" population is able to reach the sheath edge at the sample surface. 


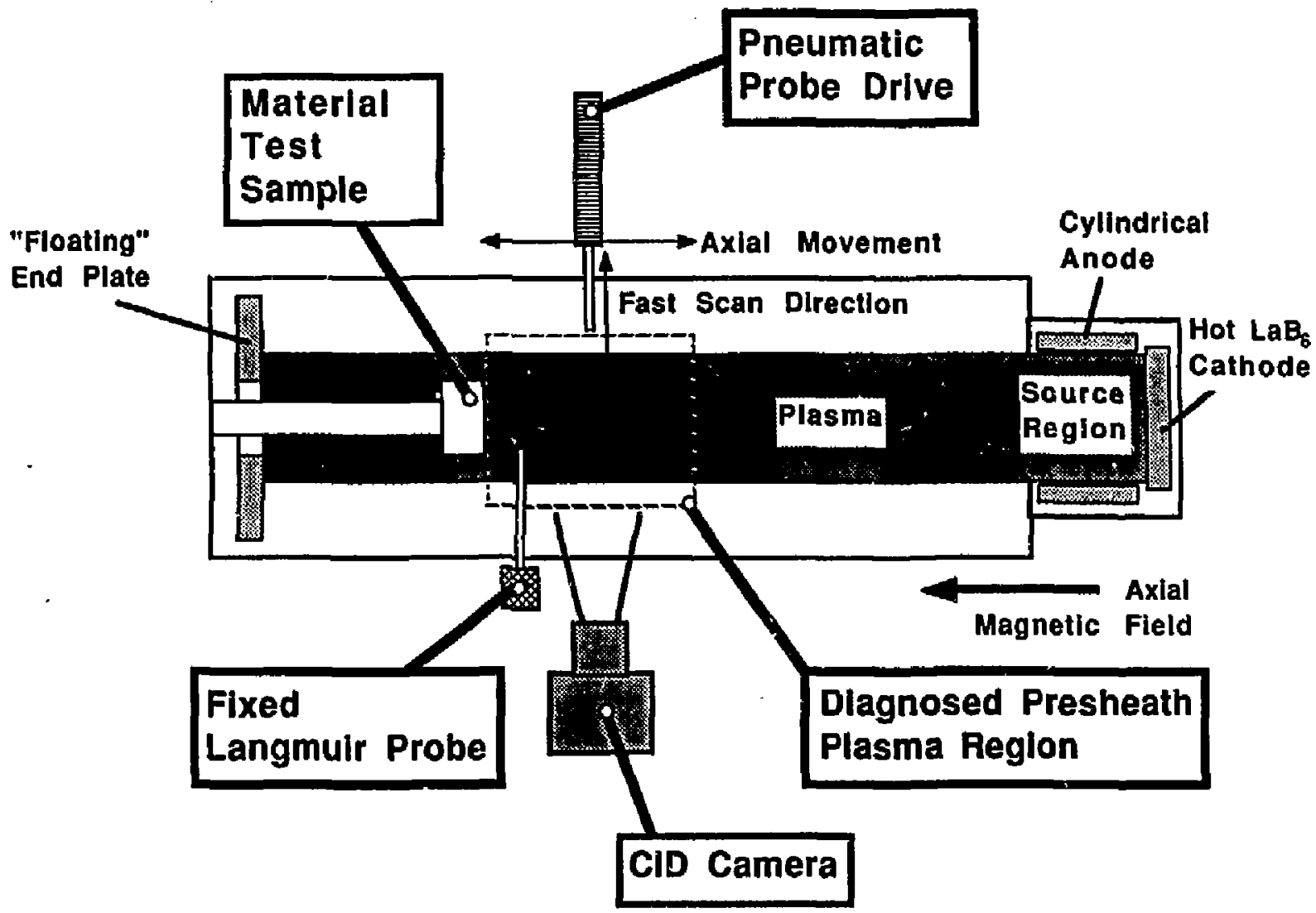

FIGURE 1 

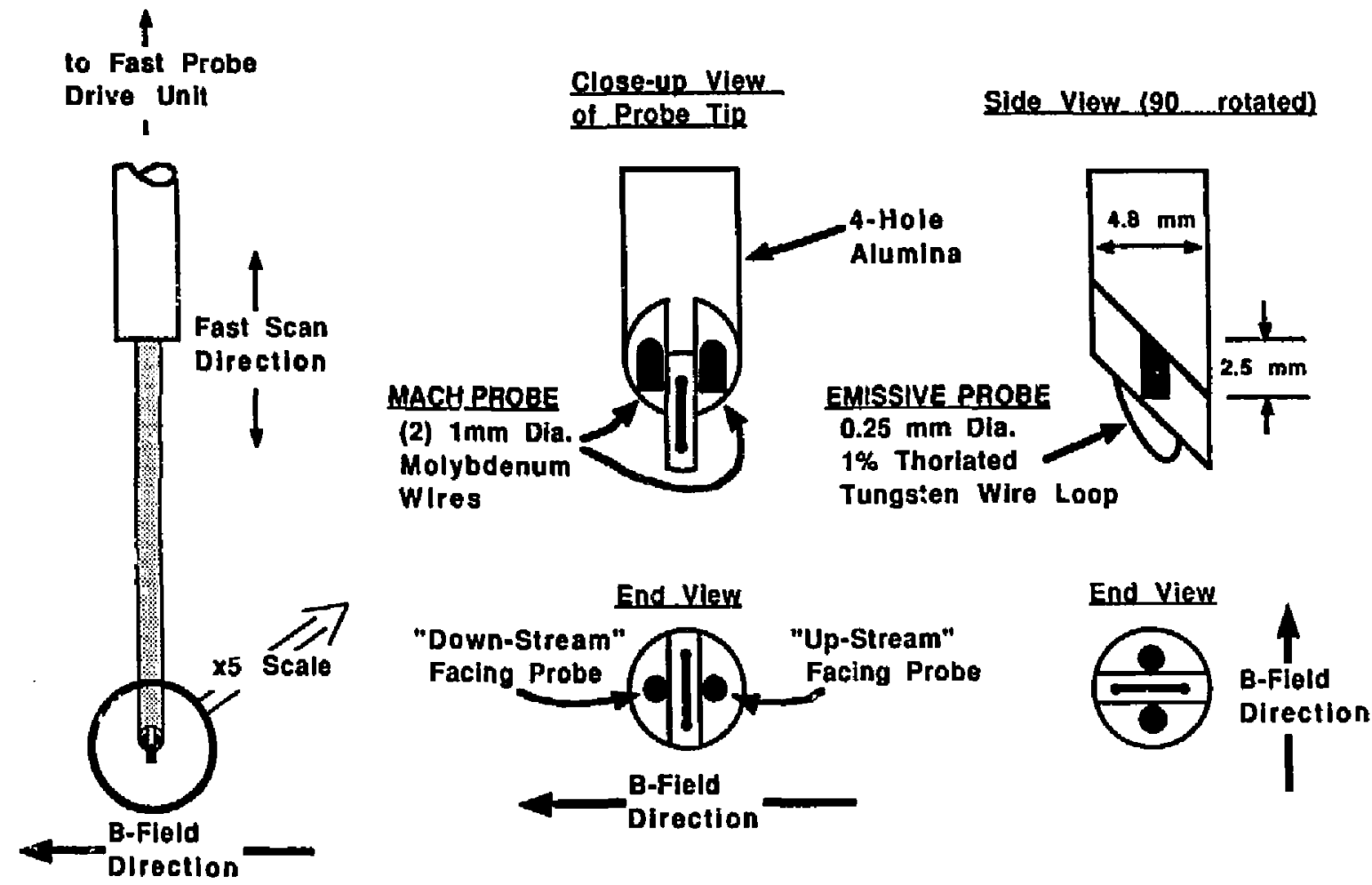
a)

Density Contours

( $3 \times 10^{11} \mathrm{~cm}^{-3}$ per contour)

Maximum $=4.4 \times 10^{12} \mathrm{~cm}^{-3}$

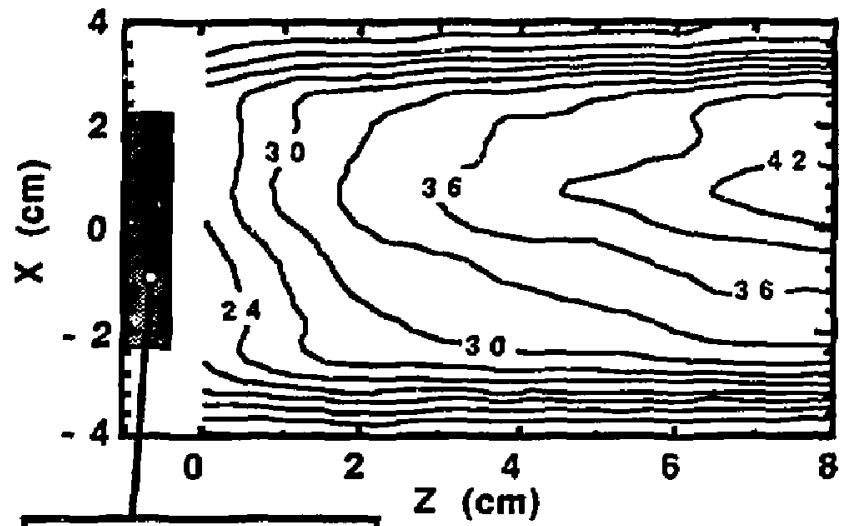

Material Sample

b) Plasma Potential Contours

(4 volts per contour)

Maximum $=33$ volts Minimum $=-12$ volts

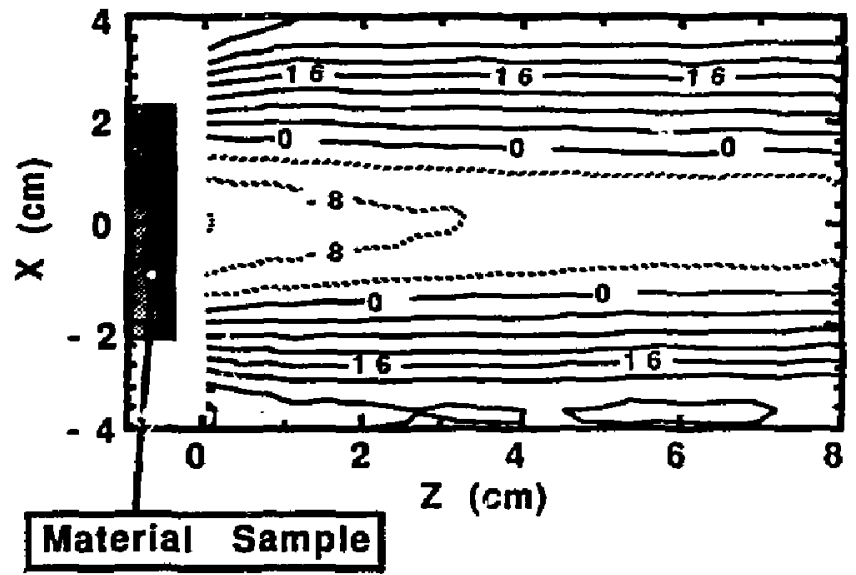

Magnetic Field

FIGURE 3 


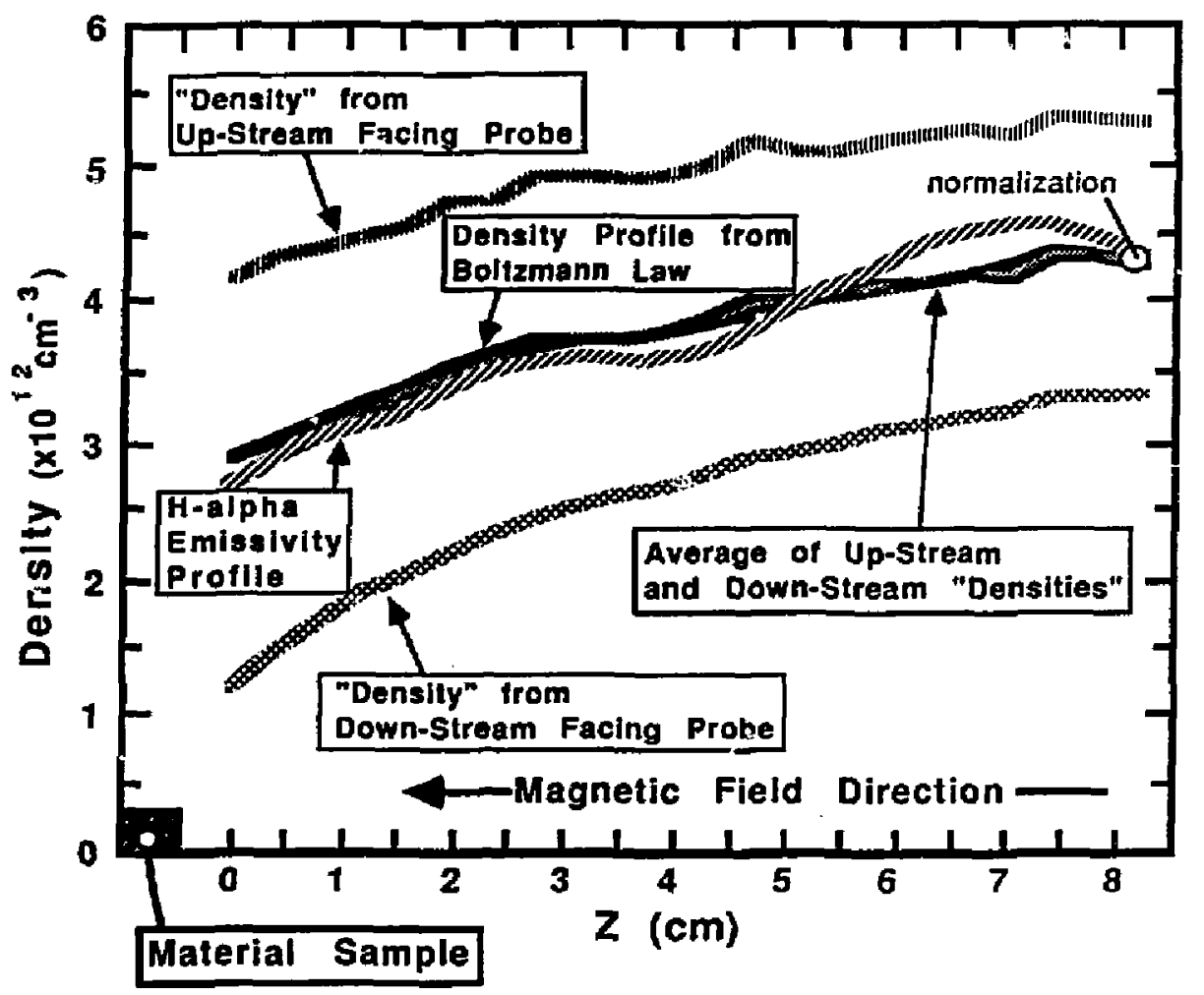

FIGURE 4 


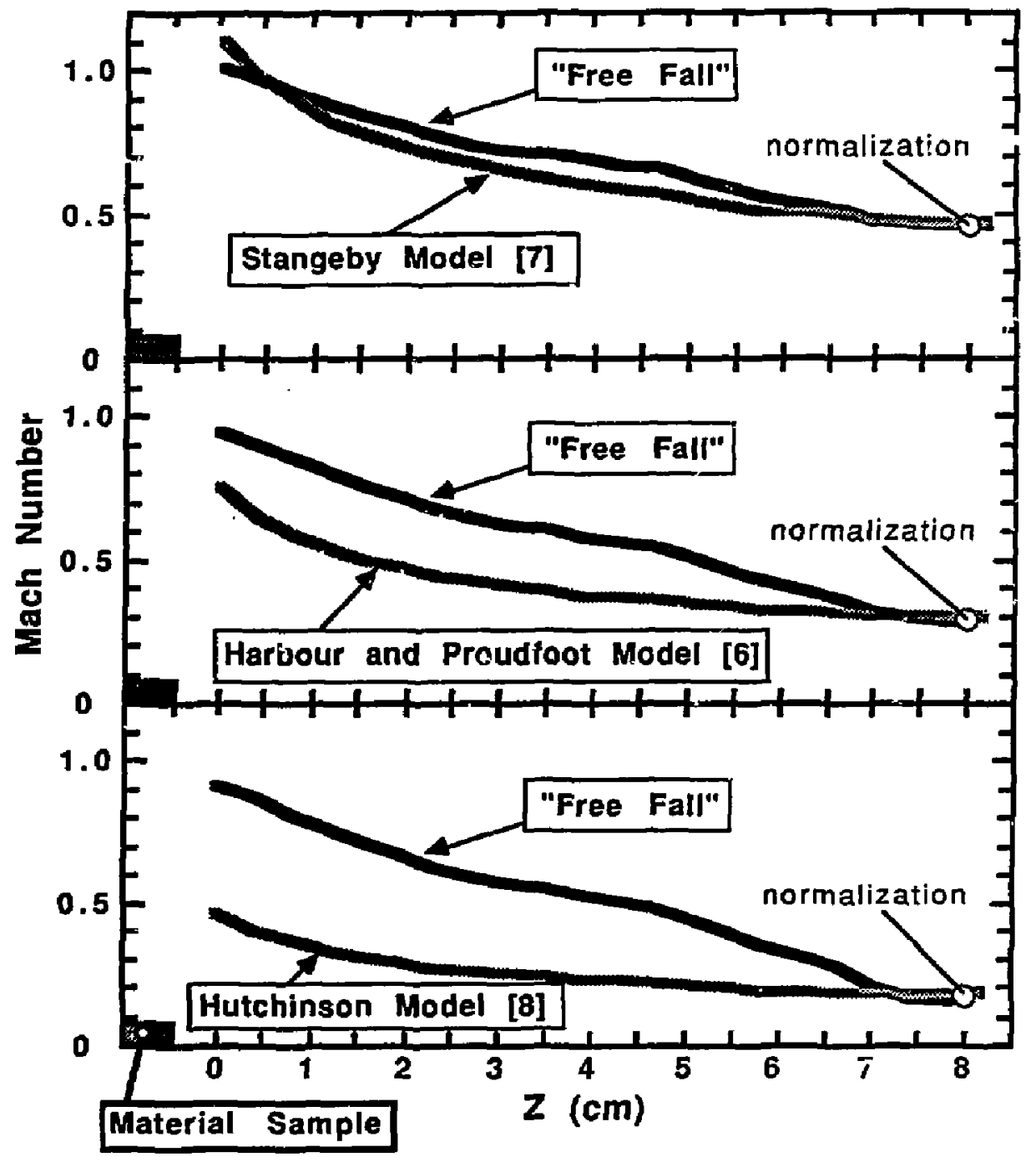

Magnetic Field Direction

FIGURE 5 


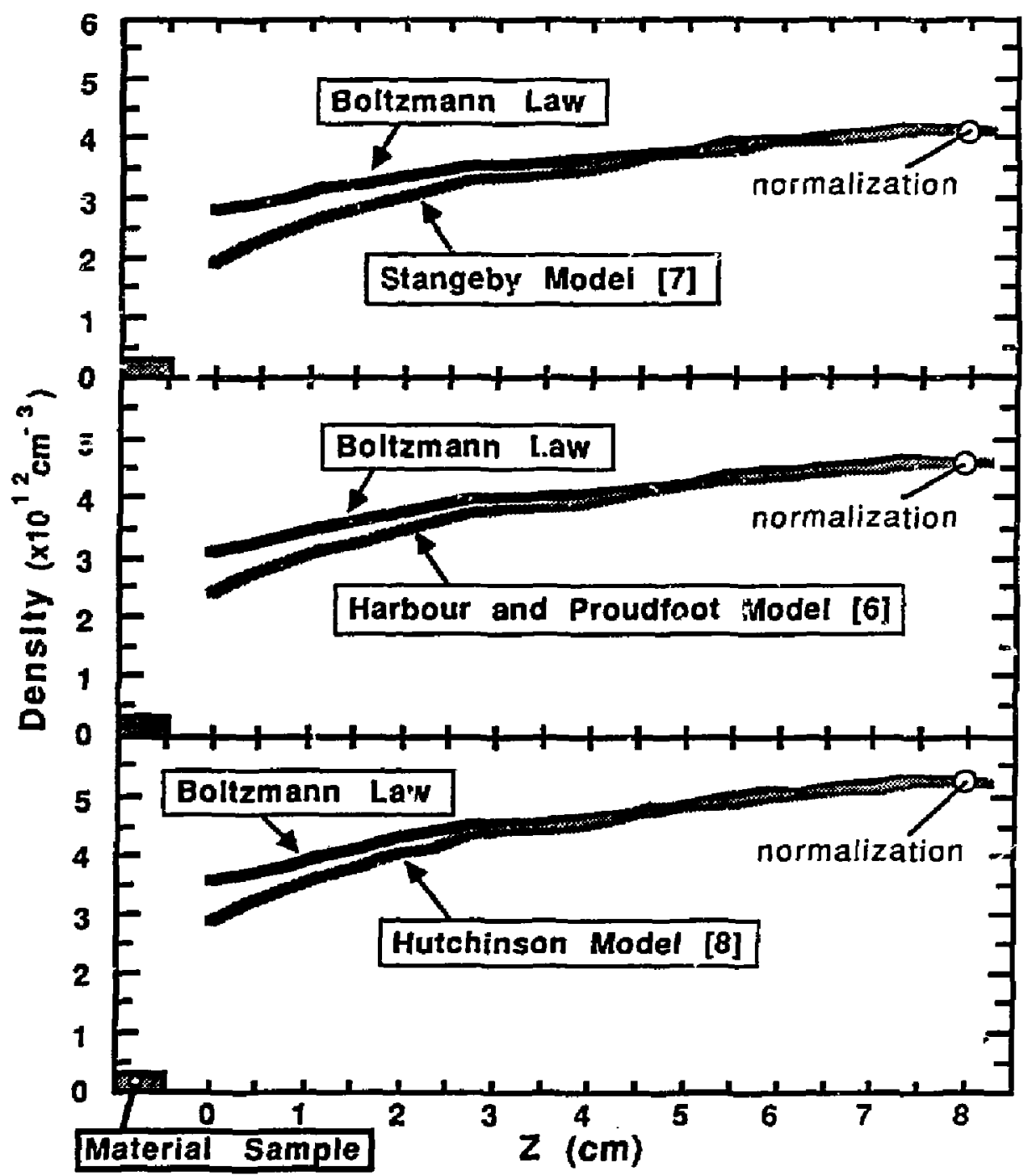

Magnetic Field Direction -

FIGURE 6 


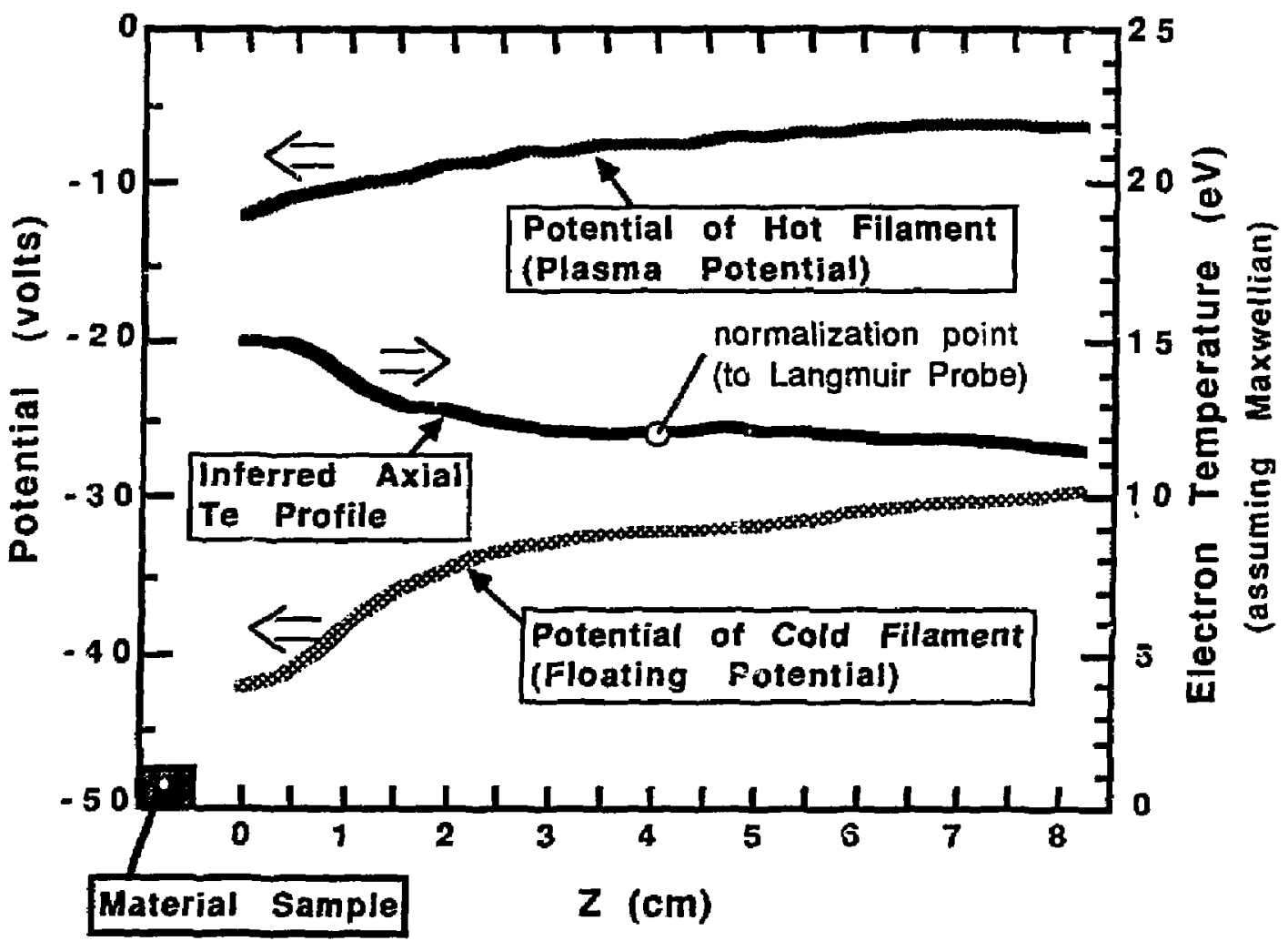

Magnetic Field Direction

FIGURE 7 